Tahmasib Kh. Fataliyev

DOI: 10.25045/jpis.v08.i2.03

Institute of Information Technology of ANAS, Baku, Azerbaijan

depart3@iit.ab.az

\title{
THE ROLE OF SOCIAL MEDIA IN THE DEVELOPMENT OF CITIZEN SCIENCE
}

\begin{abstract}
The article explores the role of social media in citizen science - a new emerging direction of e-science. It studies the development characteristics of citizen science, the activity of supporting international organizations and the role of Web 2.0 in the development of social media. The opportunities for using social media in the realization and development of citizen science are provided.
\end{abstract}

Keywords: e-science, citizen science, citizen scientist, social media, citizen science projects.

\section{Introduction}

Rapidly increasing capabilities of the information technology (IT) have given a great boost to the development of civil science, which has been shaped as a new field of e-science and characterized by the involvement of the citizens in scientific research [1]. Although the "Citizen Science" is a new term, for many years, people have been participating and collaborating in scientific research reflecting the essence of citizen science. Millions of people are involving into the study of the relationships between climate and biodiversity, the processing of the World War I agendas, the identification of new plants and animal species and continue their activities in this field.

Over the last decades, a rapid increase has been observed in the number of civilian scientific initiatives. Its scope is quite broad. There are different approaches including citizen-led projects and more mass participation initiatives led by the scientists with a large group of participants who are open to citizen society.

The interaction between scientists and people from different strata of the society is gaining steadily more recognition by the social media and researchers. The term "citizen science" is included into the Oxford English Dictionary in 2014 defined as a "study conducted by the members of the general public, typically as part of a collaborative project with professional scientists".

Generating a great deal of interest at an international level, this rapidly evolving movement in scientific activities is endorsed to a rather extensive degree. Thus, UNESCO, the European Commission and the European Environment Agency, the UK Environmental Monitoring Framework have adopted the importance of citizen science for their current activities and future political trends [2]. There are numerous reasons for this, for example, the growing demand for civil science in the fields of science, technology, engineering, the use of information collected through citizen science in formulating and implementing the policies, the studies proving the enhancing accuracy and quality of citizen science, the scope and scale of the citizen science in terms of the number of its participants and produced data.

Citizen science is characterized by the involvement of the people with different lifestyles in scientific research as the important form of public involvement in a research institution, and the citizen science leads to a new quality of scientific activity. Its scale and scope are enlarging, and therefore, the local, national and international politicians are focusing on it.

The review of the scope and scale of the people involved in the citizen science shows that its current forms have outweighed the previous forms of public involvement in research; and significant changes have taken place in the knowledge and skills of the participants. It can also be explained by the political and technological factors that stimulate the development of citizen science. It should be noted that the rapid development of education (especially higher education) during the second half of the twentieth century, including the increase in leisure time in the countries with medium and high income, and the increase in the number of educated and skilled retired people has expanded the scope of public involvement. From the technical point of view, the production, development and great opportunities of web and mobile technologies are of crucial 
importance. In addition to the development and spread of cheap sensors that can collect large amounts of data from the surroundings, the social media tools formed with Web 2.0 applications have played essential role over the past decade.

Followings can be added to above-discussed points:

- From the political perspective, it is interesting to explore the citizen science projects at the local, urban, regional, country and continental (global) levels for their geographical scope;

- Citizen science is effective due to the availability of appropriate organizational structures that can support it, especially at the local, urban and national levels;

- Recent technological advancements support new activity areas of citizen science actors and provide opportunities for the expansion of the scale of existing ones.

Taking into account all above mentioned, the article presents the study of the opportunities of social media for the formation of the citizen science, which is a new development aspect of e-science.

\section{Organizations supporting citizen science}

The Citizen Science Association (CSA), established in the United States to form a global community of civic scholars and practitioners, is the first organization supporting the citizen science [2]. The Association supports the development of citizen science through communication, coordination and education. The European Citizen Science Association (ECSA) was founded in 2013 and is coordinated by the Natural History Museum, located in Berlin, Germany. The association has members in Europe and beyond. The Association draws the attention of policy makers of the European Union and its research areas, the European Commission and its European Environment Agency and other related organizations to support the citizen science. The ECSA aims at examining the development of citizen science, particularly in the environmental monitoring of the European Union. The first international conference titled "Citizen science: open science, society and innovation in politics" was held in Berlin on 19-21 May, 2016 [4]. The ECSA announced the following ten principles of citizen science:

- Citizen science projects create new knowledge and concepts by involving citizens in science;

- Projects have realistic scientific results;

- Each professional and citizen scientist benefits from participating in projects;

- Citizen scientists can participate in several phases of the scientific process;

- Citizen scientists can get references on their participation in projects;

- Citizen science is considered as a scientific approach having limitations and directions that need to be taken into account and controlled;

- Project data and metadata are publicly available and published in open formats;

- Citizen scientists are recognized as authors in the project outcomes and publications;

- Citizen science programs are evaluated based on scientific product, quality of data, experience of participants and wider societal and political implications;

- Project managers take into account copyright and intellectual property, agreement on data exchange, confidentiality, legal and ethical issues of environmental impacts.

Another recognized organization in this field is the Australian Citizen Science Network (ACSN). The Network focuses on building a citizen science community in Australia, and held its first meeting in Australia in May 2014.

The mentioned organizations are currently creating relevant financial structures. For example, the CSA has granted everyone with free membership, and as a result, 2900 people from 65 countries have responded to this proposal. In contrast, the AVEA has chosen a paid membership model and now has more than 40 paid members who represent different organizations or are individuals. In total, 60 members attended the annual meeting of this organization. The ACSA has 
set its campaign policy, while the CSA and ACSN focus on practical activities. These organizations are holding dialogue to increase the coordination among themselves.

The experienced professionals created and developed these associations seek to promote and attain the support of government agencies to citizen science. Thus, the European Environment Agency has held a series of seminars on citizen science, and provides information on citizen science in its newsletters published in support of ACSA. The European Commission supports the activities of citizen science with the Horizon 2020 research and innovation program for the years of 2014-2020, and has set a target to provide its sustainable development.

In 2012, the European Union recognized the need for investment in citizen science and funded the projects involving the citizens in air pollution and sound monitoring, chemical, agricultural and marine water quality monitoring. Additionally, the integration of the trend "science with and for society community" within the financing scheme paves the way for new opportunities to finance the development of citizen science in the future.

At the national level, the Scottish Environmental Protection Agency provided financial support to the ACSA for the use of citizen science as a better practice. The UK Department for Environment, Food and Agriculture has recognized the importance of recent observations of cerebral diseases by citizen scientists. Indeed, the results of the observations conducted within the framework of a specific project confirmed the magnitude of the spread of this disease [5].

\section{The opportunities of social media}

The emergence and rapid development of Web 2.0 technology has greatly enhanced the possibilities of the Internet and enabled the people to connect to social networks for collaboration and exchange, regardless of their geographical location. Social networking based on this technology has become a social media that generates and disseminates unique content within a short period of time through convenient communication and file sharing. At present, social media includes blogs (Blogger, LiveJournal), micro-blogs (Twitter, FMyLife), social networking services (Facebook, LinkedIn), Wikis (Wikipedia, Wetpaint), social markers (Delicious, CiteULike), social news (Digg, Mixx), Reviews (ePinions, Yelp), multimedia sharing (Flickr, Youtube). The Facebook, Twitter, YouTube and other social media services have become an integral part of the online life of most Internet users.

Social media has a number of advantages over traditional media: social media is accessible; requires minimal expense; open to all - any person can join the global communication platform and act as a source of information; more dynamic and flexible; feedback opportunities are extensive - the audience can interact with the source of information; offers a high individualization; connects people on a single platform and enables large amounts of data exchange [6].

The range of social media users is quite diverse. Regular users benefit from social media as a tool for communication, acquaintance, daily life information, and sharing photos. Effective feedback opportunities of the social media make it a favorable communication and response channel. Recently, public authorities, political parties, civil society institutions and the private sector seek to take advantage of this potential of the social media.

The number of social networking users has grown to a considerable extent - currently, accounting for about two billion people. The number of social network users is predicted to reach 3 billion by 2020 [7]. The massive use of social networks and the availability of tools generating large amounts of information have created enormous opportunities to employ them for citizen science.

Social media is closely related to the citizen science. Thus, the citizen science projects are increasingly based on technology, and the required data is collected through online portals and mobile applications. The social element is important to motivate the volunteers to participate. Therefore, organizing forums to discuss the social media projects and sharing the results is crucial for citizen scientists. Social media has also become a tool to attract volunteers to projects and to get access to information about the project results. In addition, the statistics shows that the social 
media is not always effectively used, which can be explained by the lack of competences in proper usage of social media opportunities.

\section{Typical model of citizen science project and the role of social media in its realization}

The typical model of citizen science project can be described as follows [1]:

1. Identification of the project objectives and the scientific issues that need to be solved;

2. Forming a project team;

3. Creating, verifying and developing the protocols, data collection forms and educational materials;

4. Involving the volunteers;

5. Organizing the trainings;

6. Processing the collected data;

7. Analyzing and interpreting the obtained results;

8. Disseminating the results.

It is known that a newly emerging e-science trend, that is citizen science, is characterized by a significant number of involved volunteers. The involvement of participants in the project involves the solution of issues such as scheduling, organization of work, checking the adequacy of the collected information, processing, analyzing and disseminating the results. In the presented model, these issues constitute the basis of the project, and are of great importance for obtaining successful results. The use of social media tools in the successful implementation of each stage of the model is urgent. Hence, the collaboration technologies such as blogs, wikis, video-magazines and social networks play an important role in disseminating and sharing the research, scientific resources, ideas, information, or scientific findings for citizen scientists.

Society (community, unity) and cooperation are the key elements of many citizen science projects. Social media gives the citizen scientists the opportunity to collaborate and share research, scientific resources and outcomes [8]. For example, Pathfinder, which aims to create an online collaboration environment for citizen scientists [9]. Pathfinder has turned into education-oriented online community of educators and students working together for the development and implementation of citizen science projects. More than 20,000 users collaborate daily within the project and discuss the data protocols and catalogs, data acquisition from interactive databases and information on various research areas. Most of the citizen science projects prefer the other popular social networks (e.g., Facebook).

There are a number of well-known social media tools that is used for citizen science.

Research social networks. Research social networks (Research Gate, UniPhY, Academia, etc.) are the most popular Web 2.0 applications. Citizens and organizations have an opportunity to communicate instantly and interactively through the platforms that share information resources. Social network is the best choice for researchers working on common challenges and interests. Thus, they enable researchers to share their results, experiences and hypothesis. Social networks provide collaborative activity in their virtual lab, offering services such as communication systems, channels for sharing resources, storage and discussion forums for the research team.

Facebook. Although it is a common social network, it is profitable for its broader use for citizen science. It brings together the citizen scientists in the groups. This social network provides communication, storage, sharing and access to information.

LinkedIn. It is a platform designed to assist the people with similar profession or occupations to share information and ideas. Basically, it is intended for business users; however, it may also be useful for citizen research teams.

Twitter. Although Twitter is more microblogging than social networking, this service can be considered as a social network. It enables the users to communicate directly with their chosen partner, to track their sharing, and access information, links and documents. 
Ning. The platform allows the users to create their own social networking sites. Even though the hosting is not free, researchers widely use this well-known service to make their own private network [10].

As a consequence, by summarizing all abovementioned, it is possible to say that the social media has created the following opportunities for citizen science:

- Familiarizing the participants with the projects and each other, and forming a relevant working team;

- Fast and interactive communication;

- Sharing information on the results and developing the projects;

- Organizing and executing online training and enlightenment activities;

- Providing regular communication between the participants and stakeholders;

- Sharing information on successful results and interesting observations;

- Motivation and involvement of the participants in projects.

\section{Conclusion}

Research shows that the number of citizen science projects that have emerged as a new trend of e-science in recent years is hastily increasing. Their results are characterized by high quality. This is directly related to the development of information technologies, especially Web 2.0. The use of high-speed social media, based on these new technologies, plays an important role in promoting citizen science projects, organizing relevant trainings, involving the volunteers in projects, collecting data, discussing and distributing the results.

This work was implemented with the financial support of the Science Development Fund under the President of the Republic of Azerbaijan - Grant \# EIF-2014-9 (24) -KETPL-14/02/1

\section{References}

1. Fataliyev T.Kh. Citizen science as a new trend in the development of e-science // Problems of Information Society, 2014, No1, pp. 57-64.

2. Haklay M. Citizen Science and Policy: A European Perspective, http://www.wilsoncenter.org/publication/citizen-science-and-policy-european-perspective

3. European Citizen Science Association,http://www.ecsa.citizen-science.net

4. Citizen Science -Innovation in Open Science, Society and Policy, http://www.ecsa2016.eu

5. Chalara ash dieback in the UK,http://www.chalaramap.fera.defra.gov.uk

6. Alguliyev R.M., Imamverdiyev Y.N., Abdullayeva F.J. Social networks. "Information Technologies" publishing house, Baku, 2010, 287 p.

7. Number of social media users worldwide, http://www.statista.com/statistics/278414/numberof-worldwide-social-network-users

8. ShawJr. Edward L., Surry Dan, GreenAndre.The use of social media and citizen science to identify, track and report birds // Procedia - Social and Behavioral Sciences, 2015, №167, pp. $103-108$.

9. Luther K., Counts S., Stecher K. B., Hoff A., Johns P. Pathfinder: an online collaboration environment for citizen scientists / Proceedings of the SIGCHI Conference on Human Factors in Computing Systems (CHI '09), Boston, USA, April 04 - 09, 2009, p. 239-248.

10. Miller P. Web 2.0: Building the New Library, Ariadne İssue 45, 2005, http://www.ariadne.ac.uk/issue45/miller 